\title{
Data assimilation in a sparsely observed one-dimensional modeled MHD system
}

\author{
Z. Sun ${ }^{1}$, A. Tangborn ${ }^{2,3}$, and W. Kuang ${ }^{4}$ \\ ${ }^{1}$ Department of Mathematics and Statistics, University of Maryland-Baltimore County, Baltimore, Maryland, USA \\ ${ }^{2}$ Joint Center for Earth Sciences Technology, University of Maryland-Baltimore County, Baltimore, Maryland, USA \\ ${ }^{3}$ Global Modeling and Assimilation Office, Code 610.1, Goddard Space Flight Center, Greenbelt, Maryland, USA \\ ${ }^{4}$ Planetary Geodynamics Laboratory, Code 698 Goddard Space Flight Center, Greenbelt, MD, USA
}

Received: 24 February 2006 - Revised: 4 May 2007 - Accepted: 4 May 2007 - Published: 14 May 2007

\begin{abstract}
A one dimensional non-linear magnetohydrodynamic (MHD) system has been introduced to test a sequential optimal interpolation assimilation technique that uses a Monte-Carlo method to calculate the forecast error covariance. An ensemble of 100 model runs with perturbed initial conditions are used to construct the covariance, and the assimilation algorithm is tested using Observation Simulation Experiments (OSE's). The system is run with a variety of observation types (magnetic and/or velocity fields) and a range of observation densities. The impact of cross covariances between velocity and magnetic fields is investigated by running the assimilation with and without these terms. Sets of twin experiments show that while observing both velocity and magnetic fields has the greatest positive impact on the system, observing the magnetic field alone can also effectively constrain the system. Observations of the velocity field are ineffective as a constraint on the magnetic field, even when observations are made at every point. The implications for geomagnetic data assimilation are discussed.
\end{abstract}

\section{Introduction}

Data assimilation is a mathematical method for combining physical measurements with model output in order to get a better estimate of the state of a system. It has been applied to ocean and atmospheric systems for many years. Only recently has there been an interest in applying these techniques to other geophysical systems, such as the Earth's mantle (Bunge et al., 2002) or magnetic field (geomagnetism), which is generated and maintained by convective flow in the electrically conducting, fluid outer core of the Earth (geodynamo) (Larmor, 1919). The core convection can now be modeled numerically, albeit operating in parameter domains

Correspondence to: A. Tangborn

(tangborn@umbc.edu) far from that of the Earth (Kono and Roberts, 2002). Surface geomagnetic observations can be traced back up to 7000 years (Bloxham et al., 1989; Constable et al., 2000; Sabaka et al., 2002). Plaeomagnetic dipole moment record can be traced much further back in time (Guoyodo and Valet, 1999).

However, combining geodynamo model output with surface geomagnetic observation for better understanding of the dynamical processes in the outer core (geomagnetic data assimilation) is very different from those in other fields, primarily because of the inaccessibility to the physical quantities (or state variables) inside the outer core. The state variables necessary for modeling the core dynamics include (Kuang and Bloxham, 1999): magnetic field, which can be described by toroidal scalar $T_{b}$ and the poloidal scalar $P_{b}$; the velocity field that can also be described by similar scalars $T_{v}$ and $P_{v}$; and the density perturbation (or temperature perturbation if the core convection is driven by thermal buoyancy). It is possible that numerical models could provide more geophysical variables that may be detectable via non geomagnetic observations (Jiang et al., 2007; Kuang and Chao, 2003), but such observations and their relationship to core dynamics are still in the early stages of development.

Surface geomagnetic observations can only measure part of the poloidal scalar $P_{b}$ (e.g., if the scalar can be expanded in spherical harmonic series, the observations can only provide measurements of up to the first 14 spherical harmonic degree coefficients). These measurements can be continued downward to the core-mantle boundary (CMB) by assuming the crust and mantle are electrically insulating, approximately $3000 \mathrm{~km}$ beneath the surface. All other physical variables are practically "invisible" to surface observations.

These observational limitations present a significant obstacle to the success of any geomagnetic data assimilation system. To make progress in this direction, it is important to determine whether partially observing one variable (poloidal magnetic field) can effectively constrain unobserved variables (e.g. velocity).

Published by Copernicus GmbH on behalf of the European Geosciences Union and the American Geophysical Union. 
One could analyze the problem with a full-scale geodynamo model, such as MoSST core dynamics model we are using for related research (Kuang and Chao, 2003). But the model is very complicated: in addition to 5 fields, the model is operating in a 3-dimensional spherical shell, with 4 non-dimensional parameters describing material and thermal properties specified for the core fluid. With this approach, it is much harder to develop and test a new algorithm amid the complicated interactions between the poloidal magnetic field and other variables in the model.

A reasonable first step is to work with a simplified system which includes some important physics of the original full system, and helps us to understand the fundamental properties of geomagnetic data assimilation. The knowledge obtained from this system could then better help us navigate through the full scale geodynamo system.

Working with simplified model systems before pursuing the full scale study is very common in geodynamo studies. In the early stages of numerical dynamo modeling, simplified systems, e.g. kinematic and parameterized dynamo models (Kumar and Roberts, 1975; Roberts and Stix, 1972), had been constructed to better understand dynamo mechanisms, because of computational constraints and lack of detailed knowledge of dynamo operation. Despite the dramatic growth in computer speed and memory, computational limitations remain a problem: we do not have prior knowledge of important parameters, and data assimilation algorithms can be several orders of magnitude more expensive than simply running a geodynamo model.

The system we present in this paper is nonlinear, onedimensional (in space) with two variables. In addition to dissipation and advection, we also include coupling terms in the two equations to mimic interaction between fluid flow and magnetic field in the core. We focus on one key issue: how much can observations of one variable affect the unobserved variable (and in what direction)?

To understand this, we apply recent methodology from oceanic data assimilation systems (Borovikov et al., 2005) that share similar obstacles: satellite observations are limited to ocean surfaces, such as surface temperature and wind speed (used as boundary conditions); and a much smaller number of in situ observations are made below the surface, such as velocity, temperature and salinity. In this work, the authors used a forecast error covariance that includes cross correlation terms for the different variables, but is fixed in time. They showed that these cross correlations could be defined in a dynamically consistent manner by creating an ensemble of model runs with random external forcing. They found that calculating the covariances between different state variables was essential for the assimilation system to be successful. We propose a similar solution to reduce the computational expense of propagating error covariances in geomagnetic data assimilation.

This paper is organized as follows. The model is introduced in Sect. 2, followed by the assimilation algorithm in
Sect. 3. The results are shown in Sect. 4. In the last section, we discuss the physical implications of the experimental results and applications to the full geodynamo system.

\section{Model description}

In full geodynamo models, the governing equations include the Navier-Stokes equation for the momentum balance, the induction equation for the magnetic field, and the energy equation for density variation. Nonlinearity arises from advection of the fields (variables), and the interactions between them. These equations are solved in a spherical shell with appropriate boundary conditions (Kuang and Bloxham, 1999; Kuang and Chao, 2003).

To capture at least some of the coupling of such a system, in particular the interactions among the variables, it is useful to construct one-dimensional models. Tangborn (2004) used Burger's equation with the extended Kalman filter and Eymin (2004) used a coupled fluid-magnetic system with a fixed velocity field to test a variational magnetic assimilation system. The present work, in contrast, is a fully coupled nonlinear modeled MHD system in one-dimensional space:

$$
\begin{aligned}
& \frac{\partial b}{\partial t}=-v \frac{\partial b}{\partial x}-v \frac{\partial b_{0}}{\partial x}+q \frac{\partial^{2} b}{\partial x^{2}} \\
& \frac{\partial v}{\partial t}=-v \frac{\partial v}{\partial x}+E \frac{\partial^{2} v}{\partial x^{2}}+R b .
\end{aligned}
$$

The system is defined in the domain $[0,2 \pi] \times[0, \infty)$, and is solved with the following periodic boundary conditions and the initial condition

$$
\begin{aligned}
b(0, t) & =b(2 \pi, t), & v(0, t) & =v(2 \pi, t), \\
b(x, 0) & =f(x), & v(x, 0) & =g(x) .
\end{aligned}
$$

The initial state is generated from a long model run so as to remove and all short term transients from the initial state. This system is nondimensionalized, and the variable $b$ represents the magnetic field, $v$ represents the velocity field and $b_{0}$ describes an ambient field (specified as $b_{0}=x$ ). The parameters $q, E$ and $R$ describe the (scaled) magnetic diffusion, viscosity and the Lorentz force strength, respectively.

The velocity Eq. (2) is essentially a Burger's equation in one-dimension with a forcing term from the magnetic field. The magnetic field Eq. (1) consists of advection terms (for both the perturbed and the ambient fields) and a magnetic diffusion term. While these are model equations rather than physical equations, they can be thought of as nondimensionalized by the length scale $L=L_{o} / 2 \pi$, time scale $\tau=q L^{2} / \eta$ ( $\eta$ is the magnetic diffusivity), and magnetic scale $B_{0}$.

The simplified model does not include all of the physics of the geodynamo. However, we construct it by attempting to retain some important physics for short period geomagnetic secular variation, in particular the torsional oscillations in the 
Earth's core (Braginsky, 1976). The ocillations are governed by the balance of fluid inertia and the Lorentz force on cylindrical surfaces co-axial to the Earth's rotation axis (together with a weaker, higher order viscous damping effect). Such balance is retained in Eqs. (1), (2). Consequently, the parameters $q, E$ and $R$ of the system should be chosen carefully in numerical simulations to reflect appropriately the physics in consideration.

The variables $b$ and $v$ are discretized on the spatial grid points

$$
b_{j}=b\left(x_{j}\right), \quad v_{j}=v\left(x_{j}\right) \text { for } j=1,2, \cdots, N_{x} .
$$

The total number of grid points, $N_{x}$, is selected according to the spatial scales of the solutions. The spatial derivatives in the system (1-2) are approximated via a second order centered difference scheme. A hybrid, implicit-explicit algorithm is used for time integration of the system. The time step size varies during simulation, and is dictated by the CFL condition to ensure stability.

In time integration, the linear terms in Eqs. (1-2) are solved via a (second order, implicit) Crank-Nicolson scheme, the nonlinear terms are solved via a (second order, explicit) Adams-Bashforth scheme. When the time step size is updated according to the CFL condition, a second order RungeKutta method is used for the first two time steps before the Adams-Bashforth scheme takes over.

For easier presentation, we denote by $\boldsymbol{b}$ and $\boldsymbol{v}$ the vectors of the discrete magnetic field $b$ and the velocity field $v$ in $x$ :

$$
\boldsymbol{b} \equiv\left(b_{1}, b_{2}, \cdots, b_{N_{x}}\right)^{T}, \quad \boldsymbol{v} \equiv\left(v_{1}, v_{2}, \cdots, v_{N_{x}}\right)^{T} .
$$

The solution, or the state of the system (1-2), is defined as

$$
y \equiv\left[\begin{array}{l}
b \\
v
\end{array}\right] .
$$

Unless otherwise specified, we denote by $\boldsymbol{y}_{k}$ the state at the discrete time $t_{k}$ :

$$
\boldsymbol{y}_{k} \equiv \boldsymbol{y}\left(t_{k}\right)
$$

in the rest of the paper.

\section{Assimilation methodology}

Sequential assimilation techniques (Cohn, 1997) are a class of Bayesian methods in which a model run is used to produce a forecast (or prediction) of the state of a system. The forecast is combined with observations made at the same time in order to produce a more accurate estimate of the state, called the analysis, which in turn is used as the initial condition for a new forecast run. These methods require estimates of both the forecast and observation errors, from which an estimate of the analysis error can be made. The state of the system generally includes one or more spatial dimensions, and the errors are usually spatially correlated. Thus the errors in a discretized model are represented by an error covariance matrix. An optimal interpolation (OI) algorithm assumes that the forecast error covariance is fixed in time, and the relative weighting of the forecast and observations is determined by minimizing the analysis error.

The Kalman filter (Kalman, 1960) allows the evolution of the forecast error covariance in time using a tangent linear model (TLM). It is, however, generally intractable for large scale problems because of its excessive computational and storage requirements. The ensemble Kalman filter (enKF) has been proposed (Evensen, 1994) as an alternative to the Kalman filter which can better handle non-linearity and reduce the computational cost of evolving error covariances. Yet even this approach is computationally prohibitive for geomagnetic data assimilation, which can require a system with $8 L M N$ degrees of freedom ( $L$ is the maximum degree and $M$ is the maximum order in spherical harmonic expansion, and $N$ is the number of grid points in radius) and as many as $10^{6}$ time-steps (for a moderate truncation $L M N=40^{3}$ ) to reach 0.1 magnetic diffusive time $\tau_{\eta}\left(\tau_{\eta} \approx 200000\right.$ years for the Earth's core). For this reason we use a "frozen" forecast error covariance approximation, where the covariance is generated once using the ensemble approach. Miller et al. (1999) used an extended Kalman filter to demonstrate how properly constructed error covariances could be used to make corrections to an unobserved variable in a non-linear system. Borovikov et al. (2005) showed that a fixed multi-variate error covariance constructed from an ensemble of perturbed model runs can provide useful covariance information, particularly in regards to cross correlations between different state variables. Because changes in the Earth's core occur on very long timescales, the frozen covariance approximation may be a good approximation over the assimilation periods, which will never be more than a few hundred to a few thousand years, depending on what data sets are used.

\subsection{Ensemble calculation of a multivariate error covariance}

An ensemble of $N_{\text {ens }}=100$ model runs with perturbed initial states have been carried out, each lasting 2 time units. Denoting by $\boldsymbol{y}_{n}\left(n=1,2, \cdots, N_{\text {ens }}\right)$ the the $n$ th-simulation solution of the ensemble, the forecast error covariance matrix is then calculated as

$$
\mathbf{P}^{*}=\left\langle\left[\begin{array}{l}
\left(\boldsymbol{b}-\boldsymbol{\mu}_{b}\right) / \sigma_{b} \\
\left(\boldsymbol{v}-\boldsymbol{\mu}_{v}\right) / \sigma_{v}
\end{array}\right]\left[\begin{array}{l}
\left(\boldsymbol{b}-\boldsymbol{\mu}_{b}\right) / \sigma_{b} \\
\left(\boldsymbol{v}-\boldsymbol{\mu}_{v}\right) / \sigma_{v}
\end{array}\right]^{T}\right\rangle,
$$

where $\boldsymbol{\mu}$ and $\sigma$ are the mean and the midpoint (i.e. at the grid point $j=N_{x} / 2$ ) standard deviation of the variables over the ensemble,

$$
\begin{aligned}
\boldsymbol{\mu}_{b} & =\langle\boldsymbol{b}\rangle, \quad \boldsymbol{\mu}_{v}=\langle\boldsymbol{v}\rangle, \\
\sigma_{b} & =\sqrt{\left\langle b_{N_{x} / 2}-\mu_{\left(b, N_{x} / 2\right)}\right\rangle}, \quad \sigma_{v}=\sqrt{\left\langle v_{N_{x} / 2}-\mu_{\left(v, N_{x} / 2\right)}\right\rangle} .
\end{aligned}
$$

Equation (9) implies that the covariance matrix is scaled by the standard deviation. Similarly, we consider also the scaled 
solution

$$
\boldsymbol{y}_{s} \equiv\left[\begin{array}{c}
\boldsymbol{b} / \sigma_{b} \\
\boldsymbol{v} / \sigma_{v}
\end{array}\right]
$$

in the analysis.

The error covariance, shown in Fig. 1a, can be understood by dividing it up into blocks (sub-matrices),

$$
\mathbf{P}^{*}=\left[\begin{array}{cc}
\boldsymbol{b} \boldsymbol{b}^{T} & \boldsymbol{b} \boldsymbol{v}^{T} \\
\boldsymbol{v} \boldsymbol{b}^{T} & \boldsymbol{v} \boldsymbol{v}^{T}
\end{array}\right]=\left[\begin{array}{ll}
\mathbf{A} & \mathbf{B} \\
\mathbf{C} & \mathbf{D}
\end{array}\right],
$$

which indicates the regions of cross correlation between $\boldsymbol{b}$ and $\boldsymbol{v}$. While the $\boldsymbol{b} \boldsymbol{v}^{T}$ (or $\boldsymbol{v} \boldsymbol{b}^{T}$ ) covariance is smaller (and negative), it is still significant and indicates that observations of the magnetic field alone should have some impact on the velocity field. The small size of the ensemble (100 members vs. the 800 degrees of freedom for the model) implies that the covariance between two points that are far apart will be mostly non-physical. The covariances should therefore be truncated at large distances, and we use a piecewise continuous function (Gaspari and Cohn, 1999).

$$
\begin{aligned}
& C_{0}\left(z, \frac{1}{2}, c\right)= \\
& \begin{cases}-\frac{1}{4}\left(\frac{|z|}{c}\right)^{5}+\frac{1}{2}\left(\frac{|z|}{c}\right)^{4}+\frac{5}{8}\left(\frac{|z|}{c}\right)^{3}-\frac{5}{3}\left(\frac{|z|}{c}\right)^{2}+1, & 0 \leq|z| \leq c \\
\frac{1}{12}\left(\frac{|z|}{c}\right)^{5}-\frac{1}{2}\left(\frac{|z|}{c}\right)^{4}+\frac{5}{8}\left(\frac{|z|}{c}\right)^{3} & \\
+\frac{5}{3}\left(\frac{|z|}{c}\right)^{2}-5\left(\frac{|z|}{c}\right)+4-\frac{2}{3}\left(\frac{|z|}{c}\right)^{-1}, & c \leq|z| \leq 2 c \\
0, & 2 c \leq|z|\end{cases}
\end{aligned}
$$

to smooth the $\mathbf{P}^{*}$, where $C_{0}\left(z, \frac{1}{2}, c\right)$ is a homogeneous and isotropic correlation function. For each index couple $(j, k)$ in the matrices $\mathbf{A}, \mathbf{B}, \mathbf{C}, \mathbf{D}$, its grid point distance is $z_{j k}=\left|x_{j}-x_{k}\right|$. Then we compute the smoothing $\mathbf{P}^{*}$ by multiplying each item with $C_{0}\left(z_{j k}, \frac{1}{2}, c\right)$, given a constant $c(c=1$ in these experiments). From this we obtain the truncated covariance matrix $\mathbf{P}^{f}$, which can be seen in Fig. $1 b$.

As was shown by Gaspari and Cohn (1999), $\mathbf{P}^{f}$ is an approximation of $\mathbf{P}^{*}$ within the space limited by $C_{0}$. Covariances at distances longer than the correlation length scale are set to zero. Removal of longer correlation at larger distances, shown in Fig. 1b, effectively shortens the length scales while retaining the cross-covariances needed for multivariate assimilation. These cross-covariances are the couplings between the magnetic field $b$ and the velocity $v$ that indicate how much an unobserved variable should be corrected, given a difference between the forecast and observation $(O-F)$ in the observed variable. We will analyze the importance of this part of the error covariance by carrying out the assimilation with and without the cross covariance part of the $\mathbf{P}^{f}$ matrix in the results section.

\subsection{The sequential assimilation algorithm}

The sequential assimilation algorithm used here follows the standard optimal interpolation scheme, using the forecast er- ror covariance calculated in the previous section. At assimilation times, current observations are incorporated into the analysis, which in turn becomes the initial state for the next forecast step.

As in other data assimilation literature, in the rest of the manuscript we use the superscripts " $o$ " for observations, " $t$ " for truth, " $f$ " for forecasts, and " $a$ " for analysis. We also denote by $\boldsymbol{\epsilon}$ the errors to the state $\boldsymbol{y}$ :

$$
\boldsymbol{\epsilon} \equiv\left[\boldsymbol{\epsilon}_{b}, \boldsymbol{\epsilon}_{v}\right]^{T},
$$

where $\boldsymbol{\epsilon}_{b}$ and $\boldsymbol{\epsilon}_{v}$ are the errors for the magnetic field $b$ and the velocity field $v$, respectively.

Numerical experiments are set up using the sequential algorithm by defining an initial true state $\boldsymbol{y}_{0}^{t}$ which differs from the initial forecast $\boldsymbol{y}_{0}^{f}$ by an unbiased Gaussian distributed initial error $\epsilon_{0}$ at the initial time $t_{0}$ :

$$
\boldsymbol{y}_{0}^{f}=\boldsymbol{y}_{0}^{t}+\boldsymbol{\epsilon}_{0} .
$$

The true and model states evolve from time $t_{k}$ to $t_{k+1}$ with the same dynamics (perfect model assumption),

$$
\boldsymbol{y}_{k+1}^{t}=\mathbf{M} \boldsymbol{y}_{k}^{t} \quad \text { and } \quad \boldsymbol{y}_{k+1}^{f}=\mathbf{M} \boldsymbol{y}_{k}^{f},
$$

where $\mathbf{M}$ is the matrix arising from the spatial-temporal discretization of the system (1-2). The forecast error covariance, $\mathbf{P}^{f}$, is kept fixed in time, so that it represents more of a temporally averaged covariance rather than an instantaneous covariance. Since, by Eq. (9), $\mathbf{P}^{*}$ is scaled with respect to the midpoint standard deviations $\sigma_{b}$ and $\sigma_{v}$, and the scaled $\boldsymbol{y}_{s}^{o}$ observations are taken from the scaled true state $\boldsymbol{y}_{s}^{t}$, with unbiased Gaussian distributed errors $\epsilon_{s}^{o}$ :

$$
\boldsymbol{y}_{s}^{o}=\mathbf{H} \boldsymbol{y}_{s}^{t}+\boldsymbol{\epsilon}_{s}^{o} .
$$

The scaled analysis field $\boldsymbol{y}_{s}^{a}$ is then

$$
\left(\boldsymbol{y}_{s}^{a}\right)_{k}=\left(\boldsymbol{y}_{s}^{f}\right)_{k}+\mathbf{K}\left[\left(\boldsymbol{y}_{s}^{o}\right)_{k}-\mathbf{H}\left(\boldsymbol{y}_{s}^{f}\right)_{k}\right],
$$

where $\mathbf{H}$ is the observation operator which determines which variables and locations are observed, and $\mathbf{K}$ is the optimal gain matrix

$$
\mathbf{K}=\mathbf{P}^{f} \mathbf{H}^{T}\left(\mathbf{H} \mathbf{P}^{f} \mathbf{H}^{T}+\mathbf{R}\right)^{-1} .
$$

$\mathbf{K}$ is will be optimal under the conditions that all of the errors are Gaussian, white in time and spatially uncorrelated. It is determined by minimizing the estimated analysis error in terms of $\mathbf{K}$ and known variables (see Cohn, 1997 for details). The observation error covariance, $\mathbf{R}$, is assumed diagonal (uncorrelated observation errors) and the observations are given Gaussian distributed random errors of $1 \%$.

The unscaled analysis is determined using the standard deviations

$$
\boldsymbol{y}_{k}^{a}=\left[\sigma_{b}, \sigma_{v}\right]\left(\boldsymbol{y}_{s}^{a}\right)_{k} .
$$

The unscaled analysis solution is then used as the initial state for the next forecast. The true and forecast states used in the next section to calculate ensemble mean RMS errors as a means to test the algorithm. 


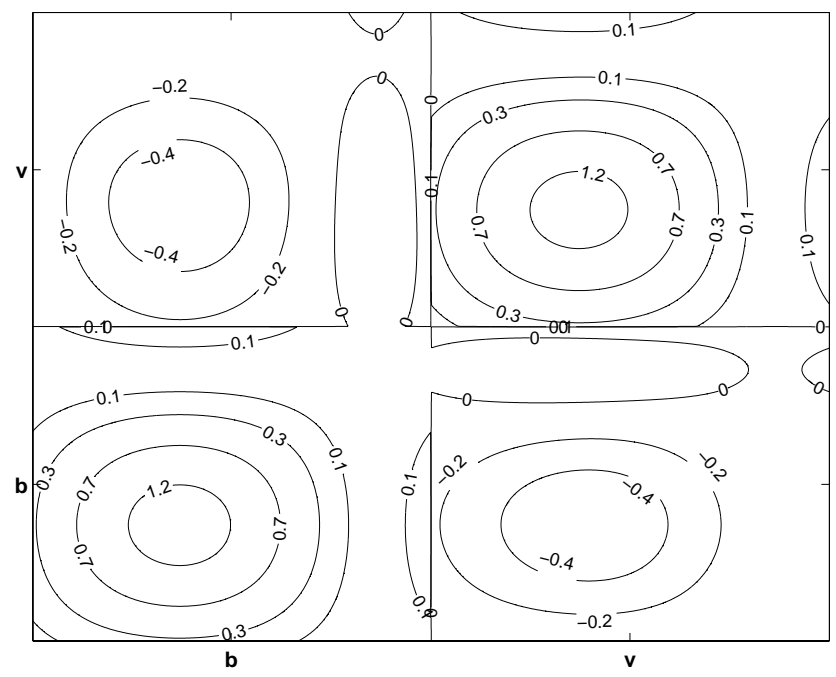

(a)

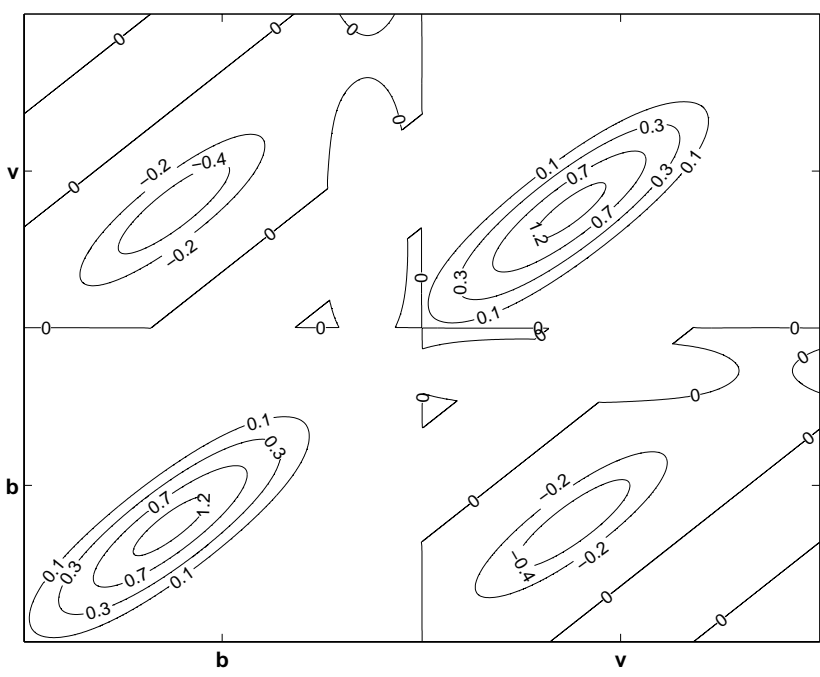

(b)

Fig. 1. The normalized forecast error covariance generated from an ensemble of 100 model runs (a), and after truncation using the GaspariCohn piecewise continuous functions (b). Note that the periodicity in the computational domain results in wrap-around of the covariances within each sub-matrix.

\subsection{Observing system simulations}

Observing system simulations have been carried out as follows: a "true" state, which uses an initial condition from well developed simulation results (those past the transient period), the forecast (or background) state from the same initial condition with an added perturbation of known Gaussian statistics that represent initial errors. In these experiments we assume no model error, so the forecast and the true state use the same numerical model. At any given time $t_{k}$, the corresponding observation is $\boldsymbol{y}_{k}^{o}$ plus some Gaussian noise $\boldsymbol{\epsilon}^{o}$ (in these numerical experiment, we control the noise's variance so that about $97 \%$ of the noise is less than $0.05 \%$ of the magnitude of the true solution $\boldsymbol{y}_{k}^{t}$ ). We are most interested in understanding how the assimilation system responds to different observing networks, including the effect of the number of observations and which of the variables are observed.

In order to approximate the limited nature of geomagnetic observations, we assume (in all but one case) that observations of the variables are made only at points much more sparse than the numerical grid points in space. The goal is to obtain some insight into the effectiveness of the Monte-Carlo constructed error covariance on the spread of the observed information to unobserved locations to and the unobserved variable. For this purpose, we have constructed the observing systems which include both $b$ and $v$ and just $b$ or $v$. In addition, we allow the observation locations vary from as many as all numerical grid points to as little as only two points in $x$. Most of the observing systems are run twice, once with the full Monte-Carlo generated error covariance, and once with the cross-covariance terms set to zero. This will help to quantify the impact of the cross-covariance terms on the non-observed variables.

\section{Assimilation results}

We have carried out a number of experiments with different parameter values and observations. In the following cases we use

$$
q=0.95, \quad E=0.01, \quad R=100,
$$

Case 1: $(\boldsymbol{b}, \boldsymbol{v})$ with 10 observations;

Case $2.1: \boldsymbol{v}$ with 10 observations;

Case 2.2: $\boldsymbol{v}$ with 400 observations;

Case $3.1: \boldsymbol{b}$ with 10 observations;

Case $3.2: \boldsymbol{b}$ with 2 observations.

The following two experiments demonstrate how the assimilation system responds with different parameter values. In these cases, ensembles of model runs with the appropriate parameter values must be run in order to generate new forecast error covariances.

Case $4.1: q=0.1, E=0.01, R=100 \boldsymbol{b}$ with 10 observations; Case $4.2: q=0.95, E=0.01, R=10 \boldsymbol{b}$ with 10 observations;

In all cases the observing networks are uniformly distributed. In order to obtain meaningful statistics, a set of $N_{e x}=20$ identical twin experiments are carried out for each observing system. The solution to Eqs. (1-4) is of the form of coupled standing waves in $\mathbf{b}$ and $\mathbf{v}$, and examples at several different times are shown in Fig. 2 using the parameter values from Cases 1-3.

We plot the mean RMS difference between the reference (or true) state and the assimilation state vs. time for each of the experiments, shown in Figs. 3-7. In each case, the initial error (or perturbation) is about $3 \times 10^{-4}$, and observation are 


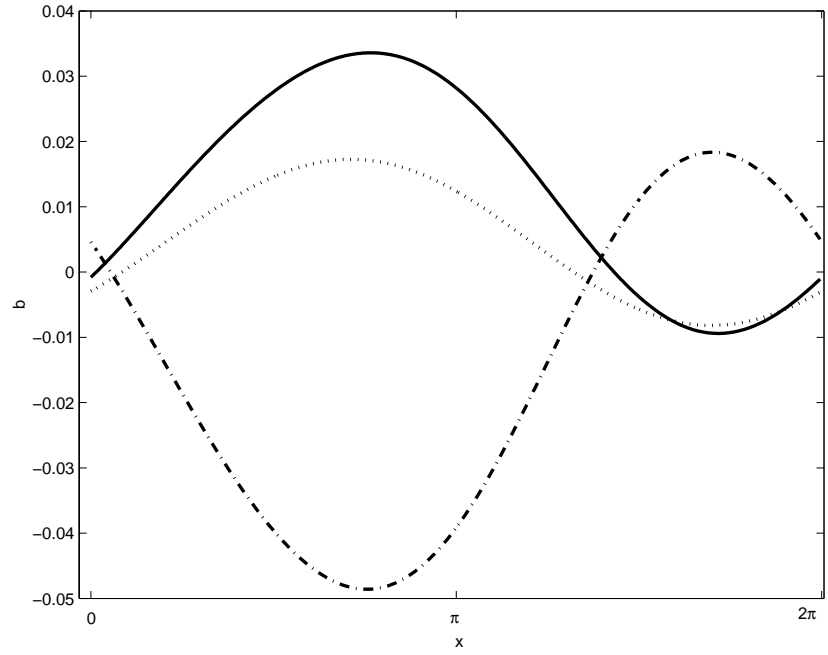

(a)

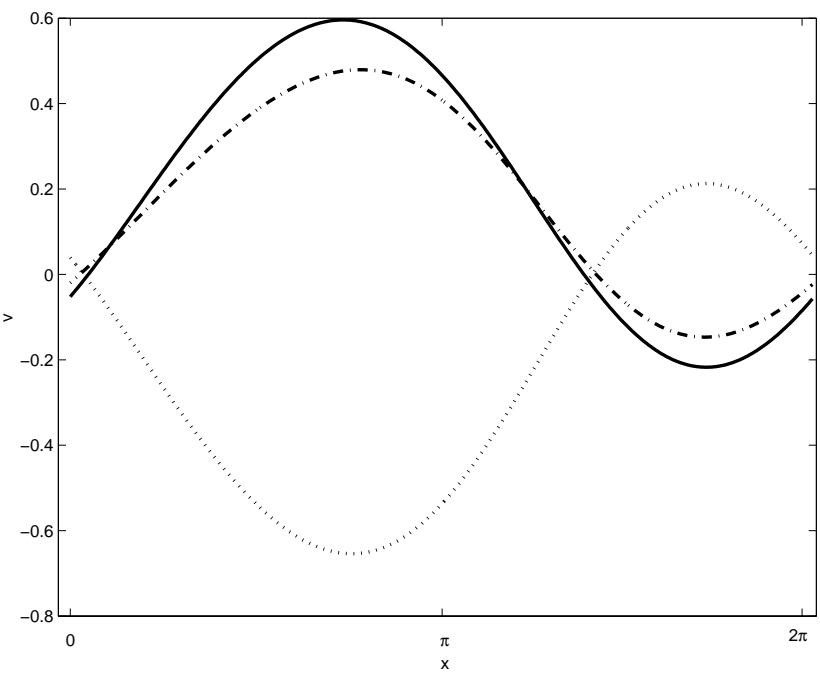

(b)

Fig. 2. Solutions to the model Eqs. (1-4) at different times, using the parameter values from cases $1-3$. These show that $\mathbf{b}$ and $\mathbf{v}$ are very nearly standing waves, with two node points.

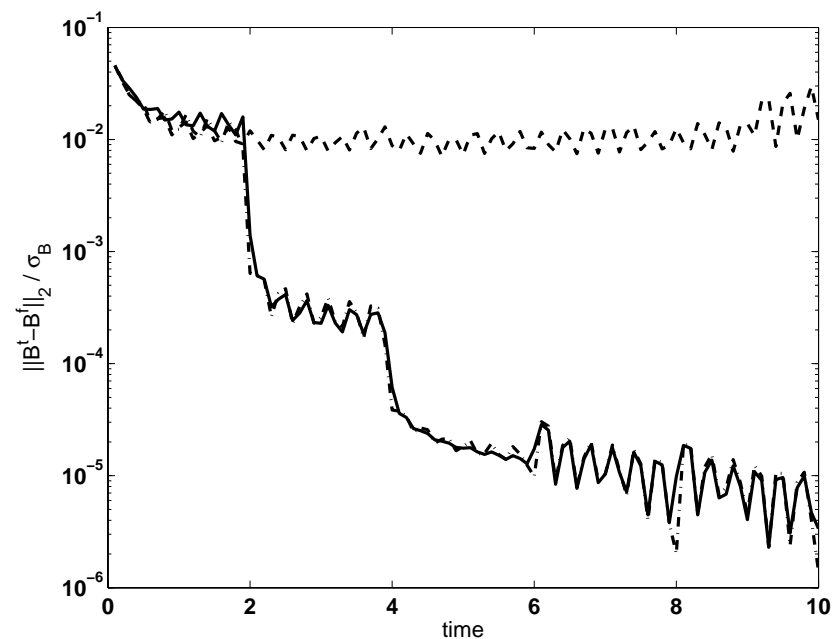

(a)

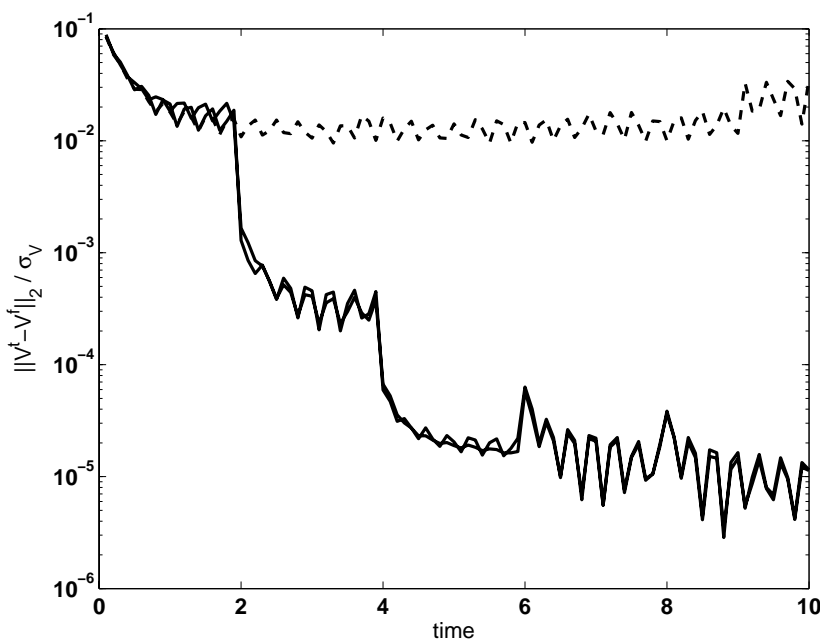

(b)

Fig. 3. Case 1, RMS error for $\boldsymbol{b}$ (a) and $\boldsymbol{v}$ (b) when both $\boldsymbol{b}$ and $\boldsymbol{v}$ are observed, at 10 uniformly spaced locations each. Assimilation is carried out at $\mathrm{t}=2,4,6,8$. The RMS error for the model run without assimilation is given by the dashed line, and with assimilation is given by the solid line. Assimilation using a forecast error covariance $P^{f}$ with the correlation between $\boldsymbol{b}$ and $\boldsymbol{v}$ set to zero (univariate) is shown by a dash-dot line. The full covariance and univariate RMS errors are essentially identically and show a significant reduction from running the model alone.

assimilated into the system every 2 time units. We calculate RMS errors for $\boldsymbol{b}$ and $\boldsymbol{v}$ using

$$
\begin{aligned}
& R M S\left(\boldsymbol{b}^{f}-\boldsymbol{b}^{t}\right)=\frac{1}{N_{e x}} \sum_{i=1}^{N_{e x}}\left[\frac{1}{N_{x}} \sum_{j=1}^{N_{x}}\left(b_{j}^{f}-b_{j}^{t}\right)^{2} / \sigma_{b}\right]_{i}^{1 / 2}, \\
& \operatorname{RMS}\left(\boldsymbol{v}^{f}-\boldsymbol{v}^{t}\right)=\frac{1}{N_{e x}} \sum_{i=1}^{N_{e x}}\left[\frac{1}{N_{x}} \sum_{j=1}^{N_{x}}\left(v_{j}^{f}-v_{j}^{t}\right)^{2} / \sigma_{v}\right]_{i}^{1 / 2},
\end{aligned}
$$

where $\sigma_{b}$ and $\sigma_{v}$ are the ensemble standard deviations defined in Sect. 3.1 and $N_{x}=400$. The difference between the forecast and truth is represented by the ensemble standard deviation, which gives a consistent normalized error for each of the two variables. Because there is no model error, a successful assimilation should gradually decrease the RMS error toward zero. In each plot, the RMS error with no assimilation is given by a dashed line, and the RMS error with assimilation using the full covariance shown in Fig. 1b is given by a solid line. We have also tested the impact of the cross covariances between $\boldsymbol{b}$ and $\boldsymbol{v}$ in these assimilations by setting them 


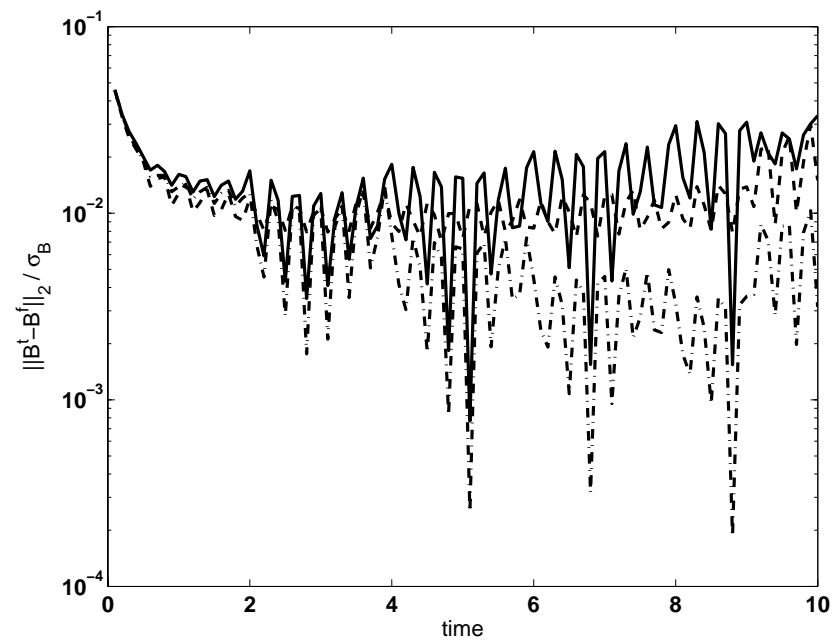

(a)

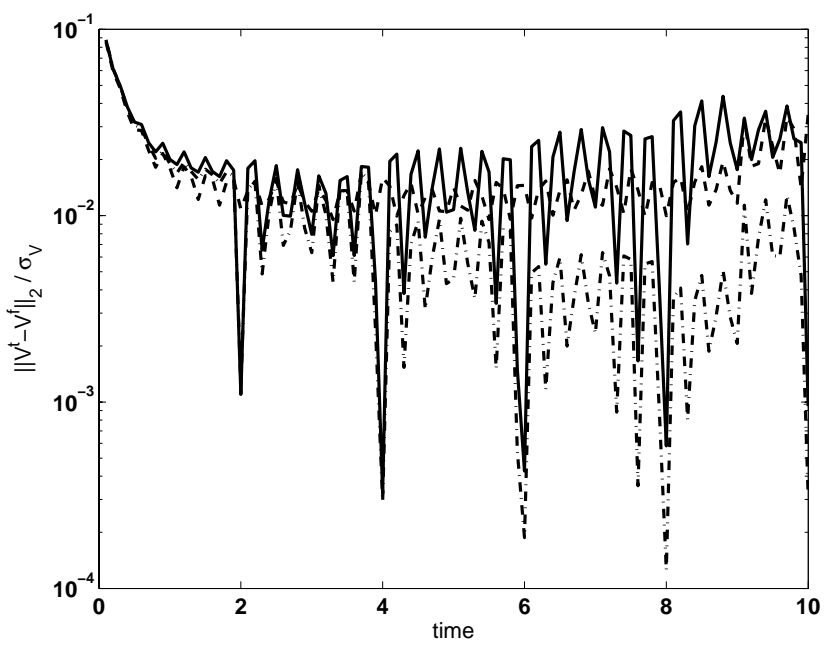

(b)

Fig. 4. Case 2.1, RMS error for $\boldsymbol{b}$ (a) and $\boldsymbol{v}$ (b) when $\boldsymbol{v}$ only is observed at 10 uniformly spaced locations.

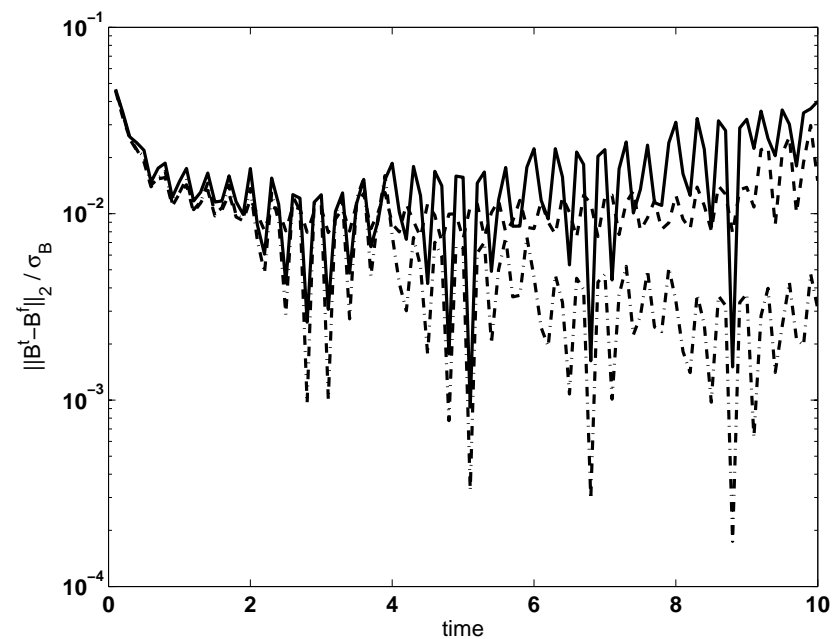

(a)

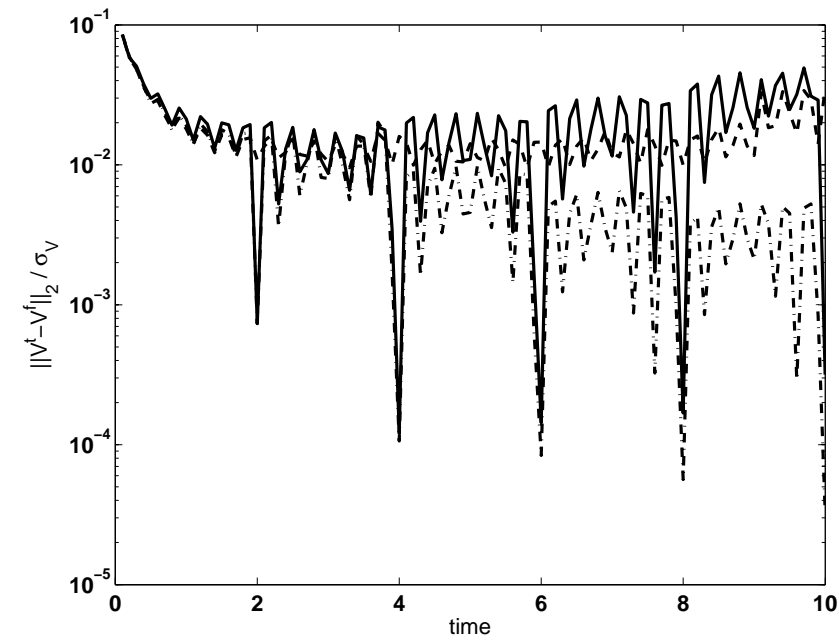

(b)

Fig. 5. Case 2.2, RMS error for $\boldsymbol{b}$ (a) and $\boldsymbol{v}$ (b) when $\boldsymbol{v}$ only is observed at 400 uniformly spaced locations. The RMS error for the model run without assimilation is given by the dashed line, assimilation with cross covariances is given by the solid line and assimilation without cross covariances is given by the dash-dot line.

to zero. The RMS error for this case is given by a dash-dot line.

In case 1 (Fig. 3), with each variable observed at 10 locations the RMS error for the forecast with assimilation decreases by nearly 4 orders of magnitude during the course of the simulation. At each assimilation time there is a noticeable drop in the RMS errors until $t=8$, when the observation accuracy is reached. There is no significant difference in the forecast errors when the cross covariance terms are removed, indicating that the correlation information is not needed when both magnetic and velocity fields are observed. It also shows that the correction is dynamically consistent as it does not produce any significant oscillations.
When only $v$ is observed in 10 places (case 2.1, Fig. 4), the assimilation is less successful. For the full covariance (solid line) there is a significant drop in the RMS error for the velocity field at each analysis time, but rapid error growth returns the error back to the pre-analysis level in a fraction of one time unit. At the same time the RMS error for $\boldsymbol{b}$ shows no drop at analysis times, which indicates that the cross covariance does not make a consistent correction to the magnetic field. The rapid rise in the velocity error is most likely due to feedback from the magnetic field, which continues to contain larger errors. With the cross covariance terms included, assimilation is actually somewhat worse than the free running model. Removal of cross covariances improves the 


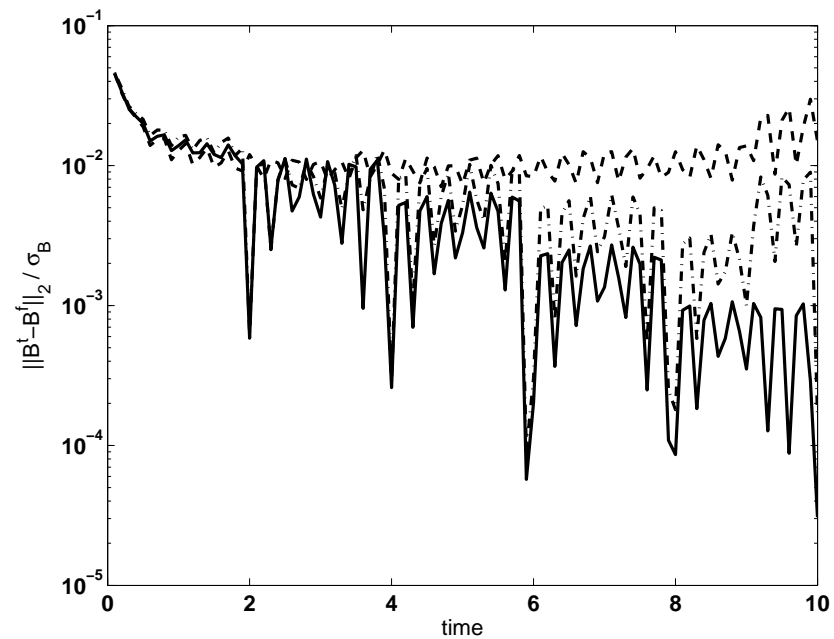

(a)

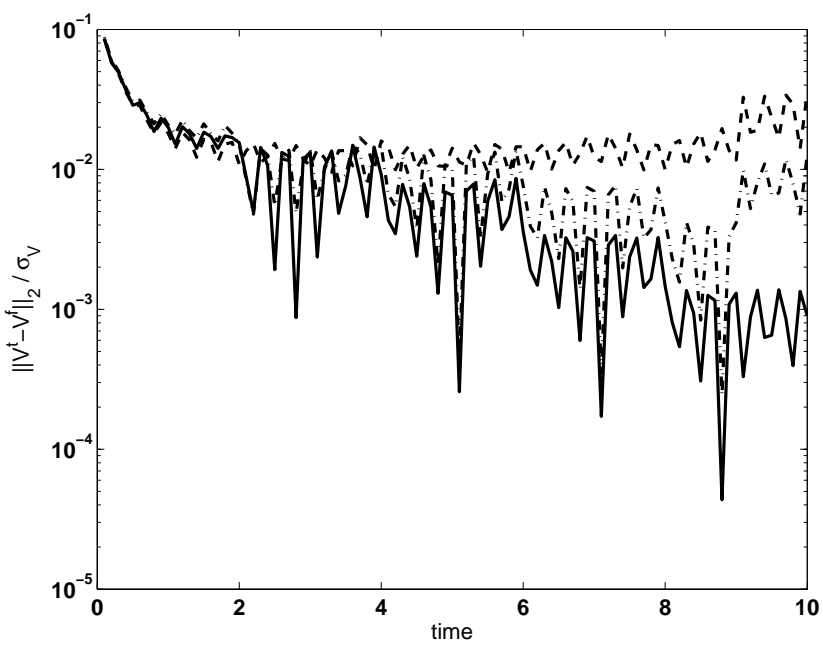

(b)

Fig. 6. Case 3.1, RMS error for $\boldsymbol{b}$ (a) and $\boldsymbol{v}$ (b) when $\boldsymbol{b}$ only is observed at 10 uniformly spaced locations. The RMS error for the model run without assimilation is given by the dashed line, assimilation with cross covariance is given by the solid line and assimilation without cross covariance is given by the dash-dot line.

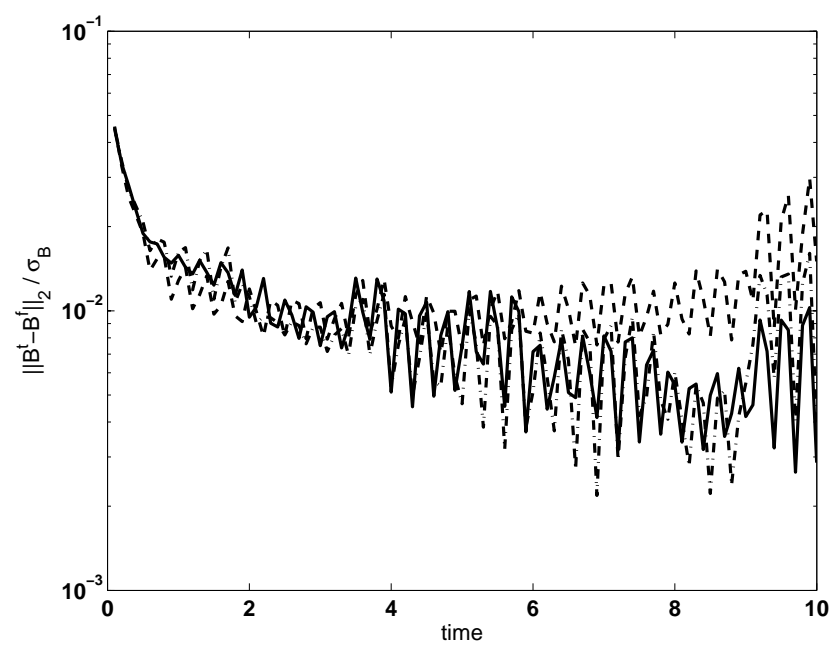

(a)

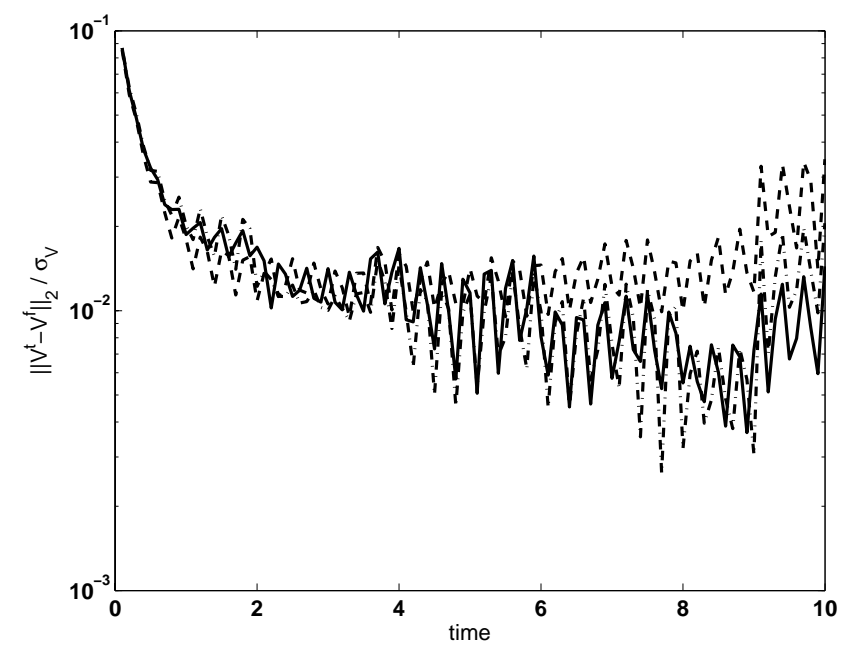

(b)

Fig. 7. Case 3.2, RMS error for $\boldsymbol{b}$ (a) and $\boldsymbol{v}(\mathbf{b})$ when $\boldsymbol{b}$ only is observed at 2 uniformly spaced locations. The RMS error for the model run without assimilation is given by the dashed line, assimilation with cross covariance is given by the solid line and assimilation without cross covariance is given by the dash-dot line.

assimilation somewhat, which again indicates that the covariance between $\boldsymbol{v}$ and $\boldsymbol{b}$ contains little or no information on how to correct the magnetic field using observations of $\boldsymbol{v}$. If the number of $v$ observations is increased to 400 (every grid point), there is no improvement (Fig. 5). This implies that there is some fundamental difference between the roles that velocity and magnetic field play in the coupled dynamics of this system.

In contrast, case 3.1 (Fig. 6) shows that when only $\boldsymbol{b}$ is observed at 10 (out of 400) points, forecast errors for both $\boldsymbol{v}$ and $\boldsymbol{b}$ are reduced significantly relative to the free running model. The importance of the correlation between $\boldsymbol{v}$ and $\boldsymbol{b}$ is seen from the drop in errors when the cross correlation is included. This indicates that there is additional information about the velocity field contained in the magnetic field observations that can only be obtained through the forecast error covariance matrix. Notice that the decrease in the RMS errors is slower than if both $\boldsymbol{v}$ and $\boldsymbol{b}$ are observed, so there is indeed information in the velocity field as well. The velocity field is just not useful by itself. The drop in errors at analysis times are smaller for $\boldsymbol{v}$ than for $\boldsymbol{b}$, implying that the frozen covariance information is not perfect. When the 


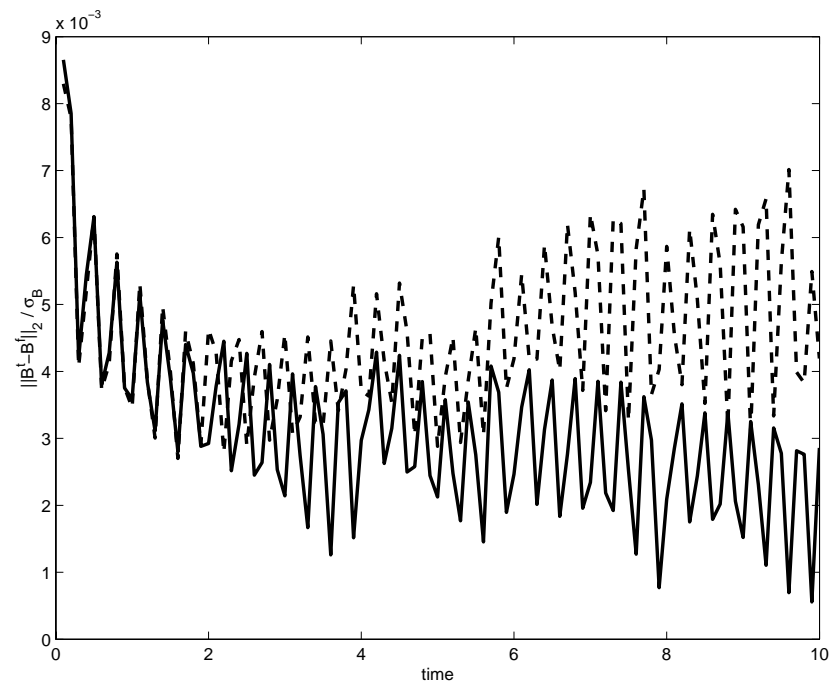

(a)

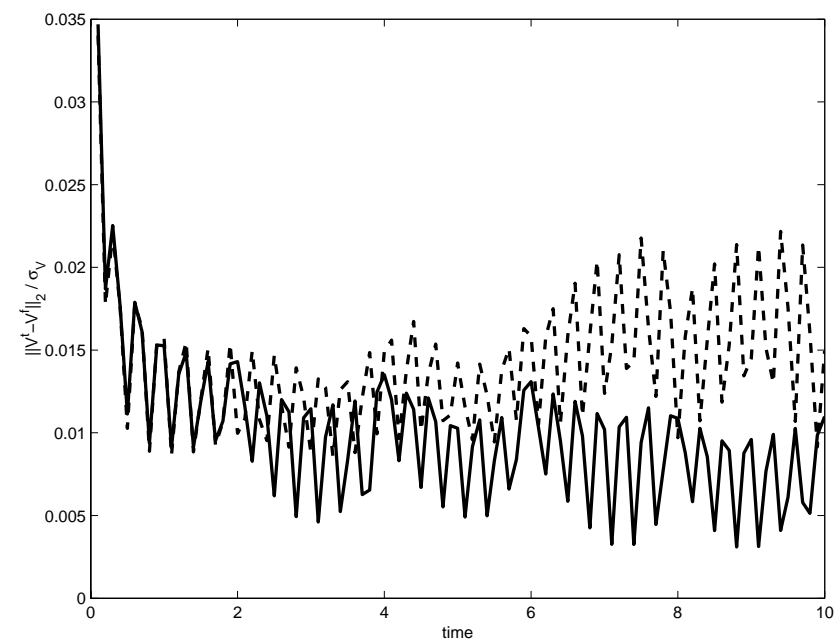

(b)

Fig. 8. Case 4.1, $R=100, q=0.1$ and $E=0.01$. RMS error for $\boldsymbol{b}$ (a) and $\boldsymbol{v}(\mathbf{b})$ when $\boldsymbol{b}$ only is observed at 10 uniformly spaced locations. The RMS error for the model run without assimilation is given by the dashed line, assimilation with cross covariance is given by the solid line and assimilation without cross covariance is given by the dash-dot line.

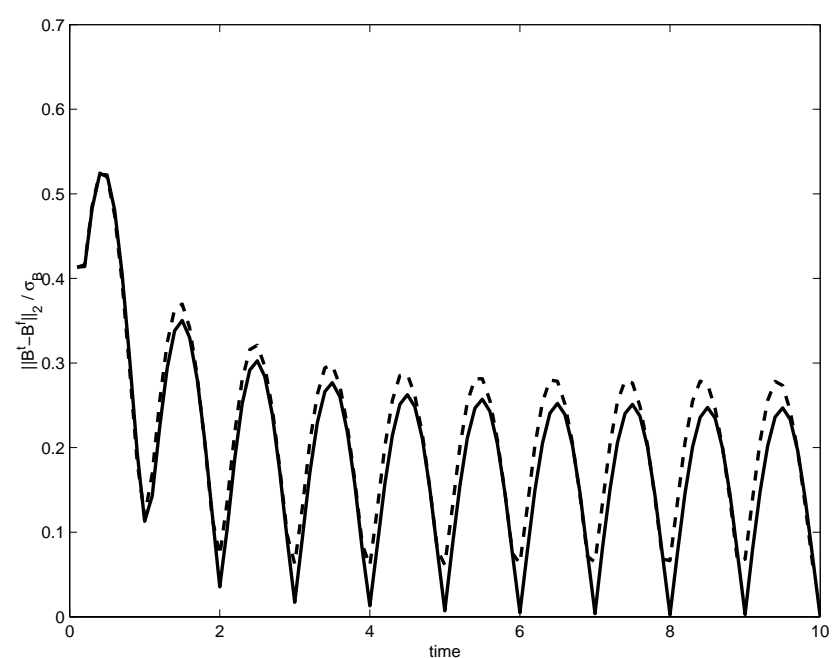

(a)

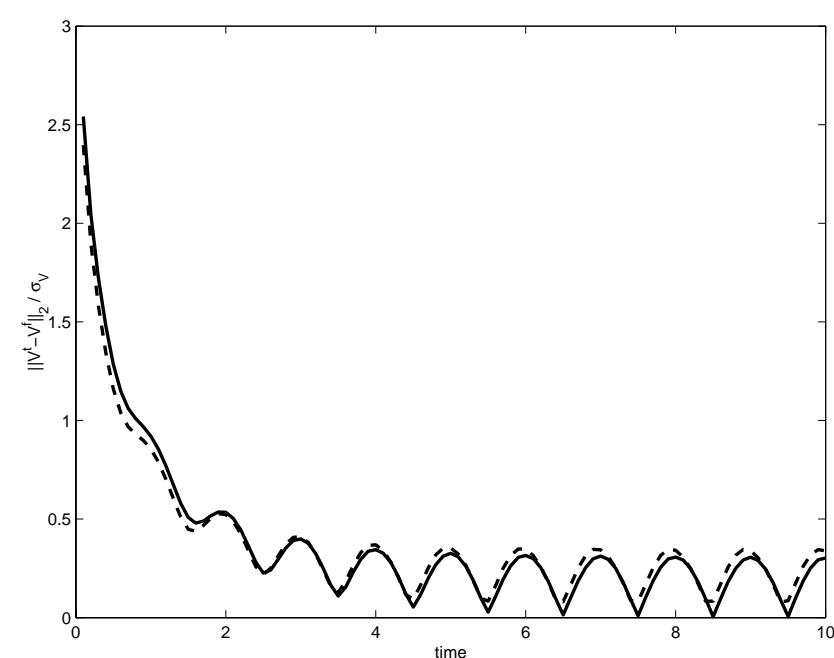

(b)

Fig. 9. Case 4.2, $R=10, q=0.95$ and $E=0.01$. RMS error for $\boldsymbol{b}$ (a) and $\boldsymbol{v}$ (b) when $\boldsymbol{b}$ only is observed at 10 uniformly spaced locations. The RMS error for the model run without assimilation is given by the dashed line and assimilation with cross covariance is given by the solid line.

number of magnetic field observations is decreased to 2 (case 3.2, Fig. 7), the rate of decrease in RMS error slows further, but the improvement due to the cross covariance term is still apparent by the end of the run.

Cases 4.1-2, (Figs. 8-9) which have the same observing network as case 3.1, show a much less dramatic drop in RMS error due to the assimilation of magnetic observations. This difference is particularly obvious in case 4.2 , where the improvement is very small with only about a $10 \%$ error reduction. This could arise from several factors, including the rela- tive importance of the coupling terms in Eqs. (1), (2) and the difference between the true state oscillation frequency and the analysis frequency.

In order to better understand these assimilation results, we have plotted the analysis errors at time $t=10$ for the ensemble mean for cases 2.1 and 3.1. Figures $10 \mathrm{a}, \mathrm{b}$ show the analysis error when the cross covariance terms are retained. For both $\boldsymbol{b}$ and $\boldsymbol{v}$, observing $\boldsymbol{b}$ results in smaller analysis errors. When the cross covariances are eliminated, the errors for $\boldsymbol{v}$ are reduced when observing $\boldsymbol{v}$. This means that the 


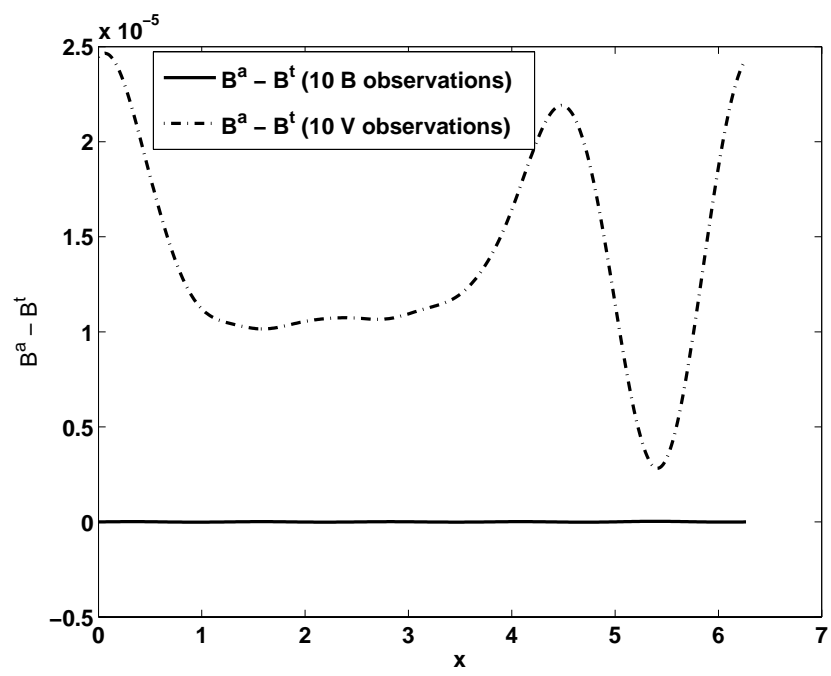

(a)

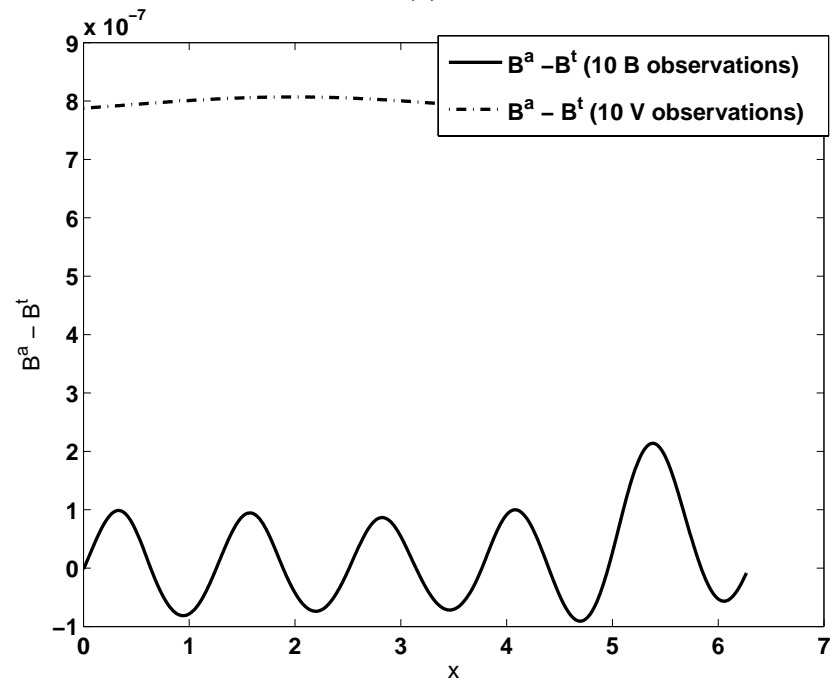

(c)

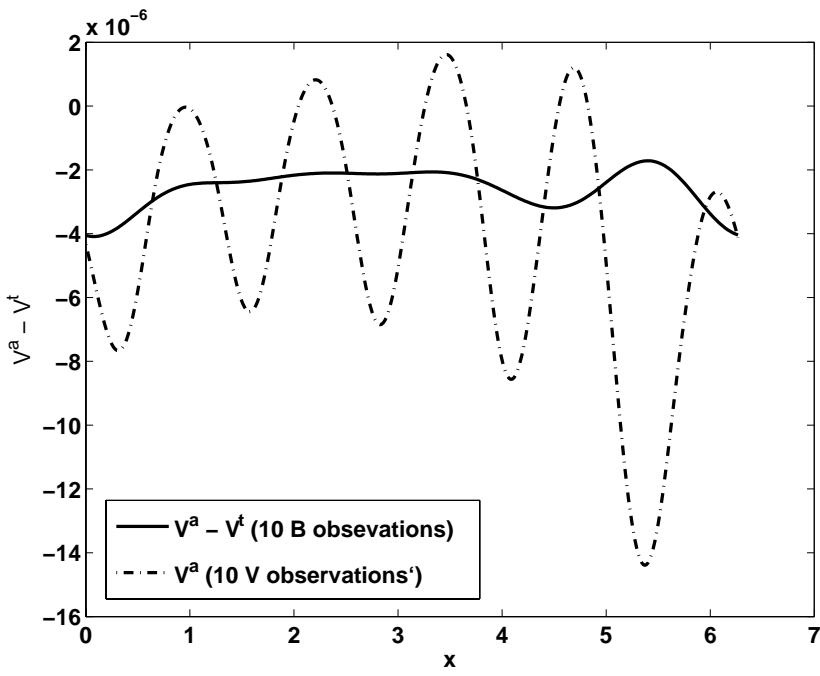

(b)

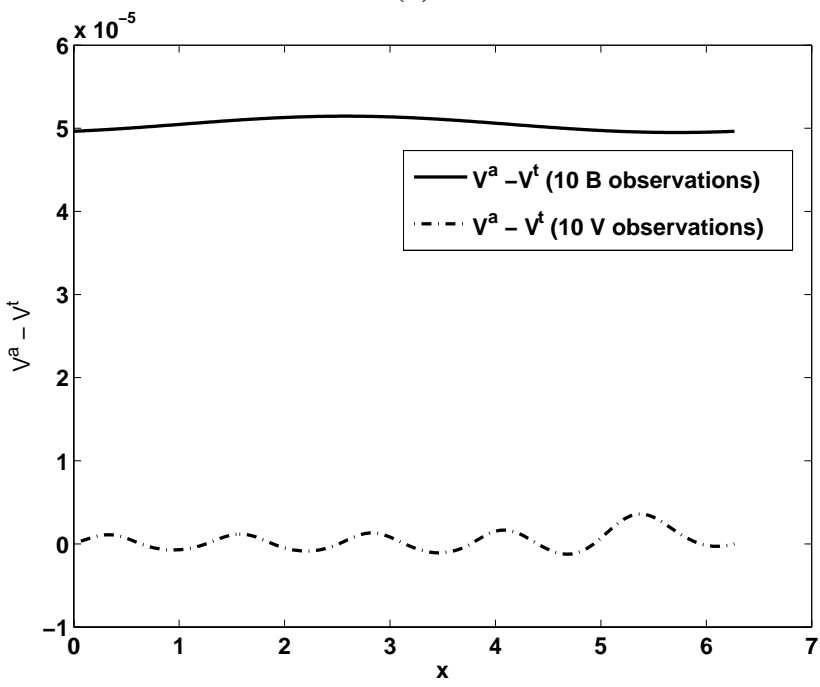

(d)

Fig. 10. Ensemble mean error at the final time with cross covariance (a) $\boldsymbol{b}^{a}-\boldsymbol{b}^{t}$, (b) $\boldsymbol{v}^{a}-\boldsymbol{v}^{t}$ and without cross covariance (c) $\boldsymbol{b}^{a}-\boldsymbol{b}^{t},(\mathbf{d})$ $\boldsymbol{v}^{a}-\boldsymbol{v}^{t}$ with 10 observations of $\boldsymbol{b}$ (solid lines) or 10 observations of $\boldsymbol{v}$ (dash-dot line). When cross covariance is retained, observing $\boldsymbol{b}$ estimates both $\boldsymbol{b}$ and $\boldsymbol{v}$ more accurately. When the cross covariance is eliminated, observing $\boldsymbol{v}$ estimates $\boldsymbol{v}$ more accurately, but $\boldsymbol{b}$ is estimated less accurately.

cross covariance terms actually degrade the analysis of $\boldsymbol{v}$. This cannot be caused directly by the coupling because there are no magnetic field observations. Rather, the analysis of the magnetic field that results is not dynamically consistent, causing more rapid growth in errors in both $\boldsymbol{v}$ and $\boldsymbol{b}$.

To understand the poor error reduction in Case 4.2, we examined the relative importance of various terms in the system Eqs. (1) and (2) and the ocillatory frequency of the true state. Analysis of the balances in Case 4.2 shows that they are similar to the other cases. For example, in the the velocity Eq. (2), the two dominant terms are the velocity time derivative and the Lorenz force, while the other terms are two orders of magnitude smaller. This dominance holds in all of the cases, though in Case 4.2, the relative importance of the advection term rises by an order of magnitude. A more significant difference occurs in the frequency of oscillation of the true state compared to the analysis frequency. These are nearly identical in Case 4.2, which likely results in a sampling error and most of the dynamics of the system being missed by the observations.

\section{Discussion}

We have carried out a series of assimilation experiments using a coupled system of nonlinear, one dimensional equations. A sequential data assimilation system was constructed 
using a frozen error covariance matrix constructed from an ensemble of model runs. Observation system experiments were set up using the same model for the true and forecast fields, differing only by an initial error (perfect model). Observation information is passed throughout the space and across fields by both advection and the error covariance.

A series of experiments were carried out with different numbers of observations. At each assimilation time, the difference between the observed and forecast magnetic field values $(O-F)$ are spread to neighboring grid points via the gain matrix $\mathbf{K}$, which depends on the error covariance $\mathbf{P}^{f}$. At the same time a correction is made to the unobserved field through the gain matrix and the cross covariance terms in $\mathbf{P}^{f}$. In addition, more distant parts of the domain are eventually affected by the assimilation by the velocity field, which advects information from the magnetic observations.

These experiments show that systematic reductions in the difference between the true and forecast fields can be achieved with as few as 2 magnetic observation locations ( $0.5 \%$ of the domain). We have demonstrated that this improvement is achieved, in part, through the cross covariance terms in $\mathbf{P}^{f}$. By contrast, observing the velocity alone at every grid point ultimately does not succeed at reducing this difference, with or without the cross covariance terms. If both velocity and magnetic fields are observed, the errors are reduced quickly to the order of the observation errors. We also see that there is less error reduction (Case 4.2) when $R$ is reduced or $q$ is increased. A direct consequence of these parameter changes is the variation of the typical time periods of the oscillations. This, coupled with the fixed analysis period for all experiments, affects the error reduction in the forecast. Case 4.2, where the analysis and oscillation frequency are nearly equal, shows the poorest performance.

Several questions arise from these experiments. Why can just two magnetic observations improve the state of the system, but a complete field of velocity measurements cannot? How might these results impact assimilation in a full geodynamo model? One reason that this transport of information only works from $\boldsymbol{b}$ to $\boldsymbol{v}$ is because the driving force of the system, $R b$ directly controls the time variation of $v$ in Eq. (1). As long as $\boldsymbol{b}$ is not identically zero, $R b$ will act to offset fluid viscosity to maintain the advection. The magnetic field $\boldsymbol{b}$ changes also from this advection. In other words, in the simplified system, $\boldsymbol{b}$ is more active, while $\boldsymbol{v}$ is more passive. From this point of view, constraining $\boldsymbol{b}$ from the observation can be much more significant than constraining $\boldsymbol{v}$.

The results from this simplified system are useful for geomagnetic data assimilation for several reasons. First, the Lorentz force in the Earth's core is very important in determining the core flow. The geodynamo is believed to be a strong field dynamo in which, aside from geostrophic flow, the Lorentz force is comparable to the Coriolis force, and to the buoyancy force in the bulk of the outer core (often called the magnetostrophic balance). This balance determines the behavior of the Magnetic-Coriolis (MC) and/or
Magnetic-Archimedian-Coriolis (MAC) waves in the core on timescales of hundreds of years and longer (Braginsky, 1967, Braginsky, 1980). On the other hand, the zonal component of the Lorenz force integrated over cylindrical surfaces co-axial to the Earth's axis (Taylor's cylinders) balances fluid inertial forces to leading order, generating torsional oscillations on decadal time scales (Braginsky, 1976). In other words, the magnetic field plays a major role in determining various flow modes in the outer core. From the experiments with the model system, we can conjecture that observing the magnetic field only should constrain the numerical geodynamo model significantly. Case 4.2 of the experiments also suggests that for successful geomagnetic data assimilation, the analysis frequency should be substantially different from the magnetic wave frequencies in the Earth's outer core.

Acknowledgements. We thank A. Fournier and the anonymous reviewer for their helpful comments on the manuscript. This research is supported by NSF Math/geophysics program under the grant EAR-0327875, and by NASA Solid Earth and Natural Hazard Program.

Edited by: O. Talagrand

Reviewed by: A. Fournier and another referee

\section{References}

Bloxham, J., Gubbins, D., and Jackson, A.: Geomagnetic secular variation, Phil. Trans. Roy. Soc. Lond. A, 329, 415-502, 1989.

Borovikov, A., Rienecker, M. M., Keppene, C. L., and Johnson, C. C.: Multivariate error covariance estimates by Monte-Carlo simulation for assimilation studies in the Pacific Ocean, Monthly Weather Review, 133, 2310-2334, 2005.

Braginsky, S. I.: Magnetic waves in the Earth's core, Geomag. Aeron., 7, 851-859, 1967.

Braginsky, S. I.: Torsional magnetohydrodynamic vibrations in the Earth's core and variations in day length, Geomag. Aeron., 10, $1-8,1976$.

Braginsky, S. I.: Magnetic waves in the core of the Earth II, Geophy. Astrophy. Fluid Dyn., 14, 189-208, 1980.

Bunge, H. P., Richards, M. A., and Baumgardner, J. R.: Mantlecirculation models with sequaential data assimilation: Inferring present-day mantle structure from plate motion histories, Phil. Trans. Roy. Soc. Lond. A, 360, 2545-2567, 2002.

Cohn, S. E.: An introduction to estimation theory, J. Meteor. Soc. Japan, 75, 257-288, 1997.

Constable, C. G., Johnson, C. L., and Lund, S. P.: Global magnetic field models for the past 3000 years:transient or permanent flux lobes?, Phil. Trans. Roy. Soc. Lond. A, 358, 991-1008, 2000.

Evensen, G.: Sequential data assimilation with a nonlinearquasigeostrophic model using Monte Carlo methods to forecast error statistics, J. Geophys. Res.-Oceans, 99, 10 143-10 162, 1994.

Eymin, C.: Étude des mouvements à la surface du noyau terrestre, $\mathrm{PhD}$ thesis, Université Pierre et Marie Curie - Paris VI, 2004.

Gaspari, G. and Cohn, S. E.: Construction of correlation function in two and three dimensions, Q. J. R. Meteorol. Soc., 125, 723-757, 1999. 
Guoyodo, Y. and Valet, J.-P.: Global changes in intensity of the Earth's magnetic field during the past 800kyr, Nature, 399, 249252, 1999.

Jiang, W., Kuang, W., Fang, M., and Cox, C.: Understanding TimeVariable Gravity due to Core Dynamical Processes with Numerical Geodynamo Modeling, in: Dynamic Planet, edited by: Tregoning, Rizos, Springer, IAG 130, pp. 473-479, 2007.

Kalman, R. E.: A New Approach to Linear Filter and Prediction Problems, ASME, Ser., D, J. of Basic Engr., 98, 35-45, 1960.

Kono, M. and Roberts, P. H.: Recent geodynamo simulations and observations of the geomagnetic field, Rev. Geophys., 40, 1-53, 2002.

Kuang, W. and Bloxham, J.: Numerical modeling of magnetohydrodyanmic convection in a rapidly rotating spherical shell: weak and strong field dynamo actions, J. Comp. Phys., 153, 5181, 1999.
Kuang, W. and Chao, B. F.: Geodynamo modeling and core-mantle interaction in the core-mantle boundary region, Geodynamics Series, 31, 193-212, 2003.

Kumar, S. and Roberts, P. H.: A three dimensional kinematic dynamo, Proc. Roy. Soc. Lond. A, 314, 235-258, 1975.

Larmor, J.: How could a rotating body such as the Sun become a magnet, Rep. Brit. Assn. Advan. Sci., p. 159-160, 1919.

Miller, R. N., Carter, E. F., and Blue, S. T.: Data assimilationinto non-linear stochastic models, Tellus, 51A, 167-194, 1999.

Roberts, P. H. and Stix, M.: $\alpha$-effect dynamos, by the BullardGellman formalism, Astr. Astrophys., 18, 453-466, 1972.

Sabaka, T. J., Olson, N., and Langel, R. A.: A comprehensive model of the quiet-time near-Earth magnetic field; phase 3., Geophys. J. Int., 151, 32-68, 2002.

Tangborn, A.: Wavelet approximation of error covariance propagation in data assimilation, Tellus A, 56, 16-28, 2004. 